\title{
Autobiographical memory and perceptual learning: A developmental study using picture recognition, naming latency, and perceptual identification
}

\author{
MARIE CARROLL and BRIAN BYRNE \\ University of New England, Armidale, New South Wales, Australia \\ and \\ KIM KIRSNER \\ University of Western Australia, Perth, Western Australia
}

\begin{abstract}
In this paper, we report four experiments aimed at extending the distinction between recognition memory and perceptual memory introduced by Jacoby and Dallas (1981). In Experiment 1, we show that dissociation of the two types of memory can be demonstrated with pictures as stimuli and with naming latency and recognition scores as responses. The depth-of-processing manipulation affects recognition but not naming, whereas both are influenced by prior exposure. Experiment 2 is a replication of the naming procedure, with a smaller set of stimuli and instructions emphasizing speed. Experiments 3 and 4 extend the paradigm to include children from 5 to 10 years old. Experiment 3 demonstrates the usual effect of depth of processing on recognition, but, in contrast to the adult data of Experiment 1, there is an analogous effect upon subsequent naming latencies. Examination of the data suggests this may be a spurious result. If not, it means either that the two memory types are not independent in children or that the "deep" subjects were implicitly naming during encoding. In Experiment 4, we test perceptual memory directly, replacing naming latency with tachistoscopic recognition. This results only in an effect of prior exposure, and not depth of processing. The results support the idea that perceptual memory is distinguishable from recognition memory, and that it is developmentally stable.
\end{abstract}

The focus of this research is an investigation of the memory processes by which adults and children determine that an item or event has earlier been encountered. In a review of their own and others' research, Jacoby and Dallas (1981) distinguished two ways in which events are remembered, ways they claimed are quite distinct from each other. On the one hand, there is a sense in which we recognize the prior occurrence of something sheerly by a sense of familiarity with its physical aspects. This they called perceptual recognition. On the other hand, there is recognition that involves recovering not only the physical aspects of an event, but also the meaningful aspects of its prior occurrence. This is the traditional recognition memory tapped in conventional memory tests. The two types of test are shown to use two different types of information for their accomplishment.

Jacoby and Dallas (1981) argued that perceptual recognition memory is revealed in "unaware" tasks, such as perceptual identification of briefly presented, previously seen, stimuli, whereas the autobiographical form of

M. V. Carroll's mailing address is: Department of Psychology, University of New England, Armidale. N.S.W. 2351, Australia. memory is revealed in the standard "aware" memory tasks, such as item recognition, in which the person is asked to effect a conscious retrieval of information.

The present research involves a test and extension of the distinction made by Jacoby and Dallas (1981): that autobiographical memory-here, recognition memoryhas to do with retrieving the unique occurrence context of an item and therefore should be sensitive to its manner of encoding during one's earlier encounter with it, whereas memory based only on perceptual fluency with the stimulus should be sensitive only to its physical characteristics and not to the context of its prior occurrence. The basic paradigm in these experiments extends the original Jacoby and Dallas paradigm in a number of ways: (1) We use pictorial, in contrast to their verbal, stimuli. (2) The perceptual fluency form of memory is tested, not by a perceptual recognition test, but by measuring picturenaming latencies for stimuli presented above recognition threshold. The choice of the picture-naming task was dictated in part by the need to use the same task for young preliterate children and adults [see (3) below]. A further question of interest was whether picture naming could be added to the list of tasks like perceptual recognition and lexical decision that show sensitivity only to the perceptual 
fluency form of memory without concurrent sensitivity to the meaningfulness of encoding. (3) The present study assesses the similarities in the performance of children and adults in their sensitivity to aware and unaware memory tasks.

Since all experiments employ the same method, a description of the paradigm is appropriate here. There were two phases to each experiment: the encoding (which varied in two ways) and the testing (which also varied in several ways). The encoding tasks varied in the type of context provided for each picture: An elaborative, deep context was provided for one group, whereas a shallow, physical context was provided for the other group. Thus, the standard levels-of-processing orienting tasks, deep and shallow, provided the variation in experiential context during encoding. Although serious criticisms have been made of levels-of-processing theory (e.g., Nelson, 1977), these are not considered relevant to this study, the sole purpose of which, in employing the levels manipulation, was to provide different kinds of encoding environments for the pictures.

In the testing phases of Experiments 1 and 3, half of those subjects who had received a shallow and a deep task were then unexpectedly given a standard recognition test, in which old and new pictures were presented for identification of prior occurrence. The other half of the subjects received a picture-naming task, in which old and new pictures were presented and latencies were measured. Such a task was expected to show only the effects of relative perceptual fluency with the old stimuli, but no sensitivity to the depth of processing manipulation. Only the strategydependent recognition task should be sensitive to context, and should show that memory for stimuli deeply encoded is better than that for stimuli shallowly encoded. In Experiment 2, only the naming task was performed at testing. In Experiment 4, subjects performed a perceptual identification task instead of recognition or naming.

By these means, we hoped to extend the generality of Jacoby and Dallas's (1981) findings. Presumably, the strongest case for the existence of these two types of memory rests on the demonstration that perceptual fluency operates through physical characteristics in general and not just through the graphemic information in words.

Several studies demonstrating a levels effect with pictorial material have been conducted. For example, Nitsch, McCarrell, Franks, and Bransford (cited in Bransford, Nitsch, \& Franks, 1977) varied participants' involvement with a picture of a living room and later tested them for proficiency at reporting details of the previously seen picture. In the shallow condition, subjects searched for inked-in Xs in the picture, although none actually were present. The deep instructions involved imagining oneself in interaction with the various objects in the room. There were large differences in recall of the picture, with shallow encoders remembering many fewer items than deep encoders. With faces, too, Bower and Karlin (1974) found levels differences between deeper tasks, such as gender classification. Thus, we expected to replicate this effect with our materials, which would permit a comparison with naming latencies, for which no deep versus shallow effect is predicted.

\section{EXPERIMENT 1}

Method
Subjects
Forty undergraduate students, males and females, were paid for
their participation in an individual experimental session lasting
30 min. Half of the subjects performed the picture-naming task,
and half the recognition memory task.

\section{Materials and Procedure}

The pictures were 90 from the set of standardized picture norms of Snodgrass and Vanderwart (1980), chosen on the basis of high picture-name agreement. The cutoff point on the picture-name agreement values was $\mathrm{H}=.37, \mathrm{H}$ being a measure of name agreement. The pictures for the encoding phase were selected in the following way: Those arbitrarily labeled numbers $1-60$ were presented to the first two subjects assigned to the deep and shallow conditions, respectively. In the test phase, these 60 , together with another 20 new ones randomly chosen from the remaining $61-90$, were presented. The 60 old pictures for successive pairs of subjects were rotated across the set of 90 , so that no picture was always old or always new. This was achieved by arranging the pictures into six groups of 15 according to their assigned number (1-90) and selecting different groups as old for each pair of subjects. Each yoked pair of subjects received the same encoding and test pictures in the same randomly arranged sequence.

The pictures were projected as monochromatic slides onto a screen by a Kodak Carousel S-AV projector. A microphone and voiceoperated relay were linked to the projector so that the subject's auditory response latency could be measured by a chronoscope from the time of onset of each slide. The picture remained on until a response had been made.

In the shallow encoding conditions, 20 subjects were instructed to observe a slide of an object and to search for an inked-in cross that might be marked somewhere on the contour of the object. On approximately one-third of all slides, a small but readily visible cross was marked. The subjects were required only to indicate its presence or absence, not its location. The subjects in the deep encoding condition were instructed to classify the object as an animate or an inanimate object; no mention was made of the significance of the crosses on some slides. Participants were allowed unlimited time to search or to classify, and no indication was given that any further test would follow. The median viewing time for searching was $1.4 \mathrm{sec}$; that for classifying was $1.2 \mathrm{sec}$.

Shortly after having viewed the slides, the subjects were told that they would be shown some further slides. Half the shallow and half the deep encoders were then given a recognition test in which they were required to say "Yes" if a slide had previously been shown and "No" if it had not. Both accuracy and response latency were measured. The remaining subjects, those in the naming task, were told that their task was now to say aloud the name of the object as quickly as possible. Most subjects later said they noticed that some pictures had been seen earlier, although they had not been told this. It was stressed that the most readily available (rather than the most appropriate) name should be the response (e.g., turtle or tortoise) and that all of the objects depicted were easily identifiable. The name and latency were both recorded.

\section{Recognition Task}

\section{Results}

Accuracy. Analysis of variance on the average proportions of correct responses shown in Table 1 yielded: a 
Table 1

Accuracy and Latency of Recognition and Naming Latencies (in Milliseconds), Experiment 1

\begin{tabular}{lrrrrr}
\hline & \multicolumn{3}{c}{ Depth of Encoding } \\
\cline { 2 - 3 } \cline { 5 - 6 } & \multicolumn{2}{c}{ Shallow } & & \multicolumn{2}{c}{ Deep } \\
\cline { 2 - 3 } \cline { 5 - 6 } & Old & New & & Old & New \\
\hline Proportion correct, Recognition & .70 & 99 & .85 & .96 \\
Median recognition latency & 1286 & 1096 & & 1075 & 1085 \\
Naming latency, mean medians & 893 & 928 & 897 & 925 \\
\hline
\end{tabular}

main old $/$ new effect $[F(1,18)=47.81, \mathrm{MSe}=.008$, $\mathrm{p}<.001]$, with hit rates being lower than correct rejections $(.77$ vs. .97); a main effect of depth $[\mathrm{F}(1,18)=5.51$, $\mathrm{MSe}=.006, \mathrm{p}<.05]$, with shallow stimuli $(.84)$ being more poorly recognized than deep (.90); and finally, a significant interaction between oldness and depth $[\mathrm{F}(1,18)$ $=9.46, \mathrm{MSe}=.008, \mathrm{p}<.01]$. Conversion to $\mathrm{d}^{\prime}$ values yielded a significant difference between shallow (mean $\mathrm{d}^{\prime}=-1.09$ ) and deep (mean $\mathrm{d}^{\prime}=-1.99$ ) encoders $[\mathrm{F}(1,18)=5.87, \mathrm{MSe}=4.99, \mathrm{p}<.02]$.

The shallow and deep encoders differed on old stimuli $(p<.01)$ but not on new. This finding, of course, confirms the original levels-of-processing studies (e.g., Craik \& Tulving, 1975), which showed that shallow encoding produces lower recognition hit rates than deep encoding. It replicates earlier work showing the effect of levels on nonverbal materials (e.g., Bower \& Karlin, 1974). More importantly, we are able to conclude that our levels manipulation was effective, so that we may assess its effects on our naming task.

Latency. The median latencies (shown in Table 1) for correct recognition responses yielded a main old/new effect $[F(1,18)=12.33$, MSe $=8074, p<.002]$, with slower responses for old (mean $=1,180 \mathrm{msec}$ ) than for new (mean $=1,090 \mathrm{msec}$ ). There was also a main effect of depth $[F(1,18)=4.12$, MSe $=248,568, p<.05]$, with responses taking longer for shallow (mean $=1,191$ msec) than for deep encoders (mean $=1,080 \mathrm{msec}$ ). There was also a significant interaction between oldness and depth $[\mathrm{F}(1,18)=14.66, \mathrm{MSe}=118,406, \mathrm{p}<.001]$; the shallow old encoders took longer than the deep, but the new did not differ.

\section{Naming Task}

The naming latencies for correct responses were analyzed. Accuracy of naming was almost perfect (fewer than $0.5 \%$ errors). A name was considered incorrect if it had not been used by any of Snodgrass and Vanderwart's (1980) subjects. Analysis of variance was carried out on the median latencies in Table 1 . Old pictures were named faster than new $[F(1,18)=15.26$, MSe $=658, \mathrm{p}<$ $.001]$, but there was no effect of depth of encoding and no interaction between depth and oldness $(F<1)$, in contrast to the recognition test. Thus, the only variable to affect naming speed was that of stimulus repetition: Name retrieval was equally fast for shallow and deep encoders, both showing facilitatory effects for old over new pictures.
This finding extends that of Jacoby and Dallas (1981), who found that a perceptual recognition task shows the same insensitivity to depth manipulations. Pictures, despite their containing physical information that makes them more discriminable from each other (more readily identifiable) than are words (Friedman \& Bourne, 1976), are insensitive to the context to the prior occurrence in a naming task.

\section{EXPERIMENT 2}

In this experiment, we did not attempt to replicate the recognition study of Experiment 1 . Although there were unequal numbers of old and new stimuli in that study, a subsequent recognition experiment using the same set of stimuli and method and the same weight judgment task (Carroll, Gates \& Roldan, 1984) had confirmed that when equal numbers of old (40) and new (40) pictures are presented for recognition, a strong depth-of-encoding effect occurs. The proportions of old stimuli reported there were .56 following shallow encoding and .91 following deep.

This experiment was simply a replication of the naming study above, conducted to conform with the procedures and methods of analysis found necessary with children.

\section{Method}

For greater comparability with the data from children reported in Experiment 3, this experiment differed methodologically from the naming section of Experiment 1 in the following ways: First, 32 subjects, young adults who had not participated in Experiment 1, were urged to name the pictures as fast as possible. Speed only was stressed above all, rather than accuracy and speed as above. Second, equal numbers of old and new stimuli $(25$ each) were presented for test, and 25 were presented for the orienting task. Third, the deep orienting task was changed to that employed in Experiment 3; instead of judging animacy, these subjects now judged the weight of the object and estimated whether they could pick it up and carry it.

\section{Results and Discussion}

The mean median naming latencies were: shallow encoding-old, $783 \mathrm{msec}$, and new, $833 \mathrm{msec}$; deep encoding-old, $789 \mathrm{msec}$, and new, $822 \mathrm{msec}$. The data support the findings of Experiment 1-an effect of prior exposure [old/new: $\mathrm{F}(1,30)=20.5, \mathrm{p}<.01, \mathrm{MSe}=$ $1,068]$, but no additional advantage for deep encoders (the old/new difference for "deep" subjects is actually slightly less than that for "shallows"). As expected, there was no main effect of depth $[F(1,30)=.03]$. The speed instructions apparently had their desired effects, since the overall times were below those for Experiment 1.

\section{EXPERIMENT 3}

Using pictorial tasks, Experiments 1 and 2 supported the distinction made by Jacoby and Dallas (1981) between autobiographical memory and perceptual learning. One important advantage of using pictures as stimuli is that the investigation of these memory forms can now be ex- 
tended to nonliterate subjects, including young children. In Experiments 3 and 4, we report what we believe are the first studies of perceptual memory in children. The background literature provides only a broad guide to expectations in this case. Perhaps the firmest foundation is furnished by the well-accepted distinction between remembering that is based on the use of deliberate, mnemonic strategies and remembering that is done automatically (Brown, 1973; Flavell, 1977; Hasher \& Zacks, 1979). It is generally claimed that the former type of remembering shows improvement with age, whereas the latter is developmentally invariant, or relatively so. Insofar as perceptual memory, of the sort that Jacoby and Dallas (1981) had in mind and that was further demonstrated in the first two experiments, is seen as an unaware form of memory, it can hardly be grouped together with the deliberate or effortful memory processes. As a first approximation, therefore, perceptual memory can be regarded as an automatic process, and we are led to predict developmental invariance.

Hence, in Experiment 3, we tested children ranging from 5 to 10 years of age in an attempt to test this prediction.

It is important to acknowledge, however, as a reviewer of an earlier version of this paper pointed out, that confirming developmental invariance would not prove that perceptual memory is in fact an automatic process. It would merely fail to disprove this contention, and would add plausibility to it.

In the course of looking for evidence of perceptual memory in childhood, it was necessary to adopt the logic of previous experiments, namely, that of seeking a dissociation between recognition memory and perceptual memory. Thus, we again had subjects undertake a shallow or deep orienting task (searching and classifying) and then either attempt to recognize the old items in an enlarged set or to name the old and new items as quickly as possible. This procedure also meant that we were collecting developmental data on recognition memory itself. Although this was not the focus of the research, it is worth pointing out that there is debate about whether recognition is developmentally stable (Brown, 1975; Olson, 1976; Piaget \& Inhelder, 1973) or not (e.g., Dirks \& Neisser, 1977; Mandler \& Robinson, 1978; Sophian \& Stigler, 1981). One might imagine that data would simply decide the matter-that if developmental trends emerged, recognition could be said to improve with age. But as Sophian and Stigler (1981) pointed out, improvement may be due to changes in other processes (verbal skills, decision criteria, and so on), whereas recognition per se remains fixed.

Recognition can of course be influenced by orienting activity, with deep encoding leading to better performance than shallow. Thus, it may be the case that age-related changes in recognition can be attributed to greater spontaneous use of elaborated encoding at input by older children, as has been suggested for recall (Geis \& Hall, 1976). If this were true, we could expect the elimination of age differences when children are forced to encode in fixed ways. Our design permits us to comment on this proposition.

\section{Subjects \\ Method}

The subjects were three groups of children: 5-year-olds, 7-yearolds, and 10-year-olds ( $n=42$ per group). Most were selected from the same elementary school and from the same classes for each age group. The major focus of the study was naming latencies, and 26 or each age group participated in that part of the experiment. Of them, half (13) were assigned to the shallow and half to the deep encoding conditions. The remaining 16 children of each age received the recognition task, 8 following the shallow orienting task and 8 the deep.

\section{Materials and Procedure}

These followed the format of Experiment 2, with searching for a cross marked on the drawing's outline being the shallow task, and judgments of portability being the deep task. Twenty-five old and 25 new slides were presented in Phase 2 (rapid naming or recognition) of the experiment, which followed a study phase of 25 stimuli.

Testing was carried out individually in a small private area within the school. The children were allocated to search or classify and then were given an unexpected recognition or naming task. Each session lasted from 10 to 25 min. During the testing phase, when responses and latencies were being measured by means of a voiceactivated relay, it was necessary for one experimenter to sit beside the 5-year-olds and constantly encourage them to respond quickly and to speak into their hand-held microphones. The experimenter who did this was blind to the particular set of stimuli designated old and new and to the encoding condition. For the older groups, the instruction to respond with speed and accuracy was sufficient if repeated only occasionally. All subjects appeared to cope well with the tasks of detecting crosses or judging heaviness.

\section{Results \\ Recognition Memory: Accuracy}

Correct detection rates for old and new stimuli are shown in Table 2. It is obvious that rejection rates were nearly perfect for all age groups, and they are not analyzed further. For the old data, there are significant age $[F(1,42)=16.19, \mathrm{p}<.001]$ and depth $[\mathrm{F}(1,42)=70.74$, $\mathrm{p}<.001]$ effects. The age means are: 5 -year-olds, .51; 7-year-olds, .78; and 10-year-olds, .83. The shallow mean is .51 , and that for deep is .91 . The interaction of age and depth was not significant $(F=.88)$.

The data were converted to $d^{\prime}$ values (see Table 2 ) and submitted to analysis of variance. There were significant effects of age [mean d's $=-.58,-2.42$, and -2.59 for ages 5,7 , and 10 , respectively; $F(2,42)=11.89$, MSe $=1.67, \mathrm{p}<.001]$ and depth [mean $\mathrm{d}^{\prime} \mathrm{s}=-.66$ and

Table 2

Proportions Correct in Recognition and d', Experiment 3

\begin{tabular}{cccccccc}
\hline & \multicolumn{5}{c}{ Depth of Encoding } \\
\cline { 2 - 4 } Age of & \multicolumn{2}{c}{ Shallow } & & \multicolumn{2}{c}{ Deep } \\
\cline { 2 - 7 } Subject & Old & New & & d' & Old & New & d' \\
\hline 5-year-olds & .28 & .96 & .48 & .76 & .93 & -1.65 \\
7-year-olds & .60 & .98 & -1.35 & .97 & .99 & -3.49 \\
10-year-olds & .66 & .98 & -1.09 & .99 & 1.00 & -4.10 \\
\hline
\end{tabular}


-3.08 for shallow and deep, respectively; $F(1,42)=$ $42.02, \mathrm{MSe}=1.67, \mathrm{p}<.001]$, but no interaction of age and depth $(\mathrm{F}=.59)$.

\section{Recognition Memory: Latency}

Latencies for correct responses yielded a main effect of age $[\mathrm{F}(2,42)=22.63, \mathrm{MSe}=133,238, \mathrm{p}<.001]$, with decreases in time as the age increased (means = $1,666,1,354$, and $1,052 \mathrm{msec}$ for ages 5,7 , and 10 , respectively). The oldness effect $[F(1,38)=20.57$, MSe $=15,726, \mathrm{p}<.001]$ showed that old stimuli were responded to more slowly (mean $=1,415 \mathrm{msec}$ ) than new $($ mean $=1,299 \mathrm{msec})$. The median latencies presented in Table 3 show the interactions between age and oldness $[\mathrm{F}(2,38)=6.53, \mathrm{MSe}=15,726, \mathrm{p}<.005]$ and depth and oldness $[\mathrm{F}(1,38)=7.88, \mathrm{MSe}=15,726, \mathrm{p}<.01]$. The 10-year-olds showed no overall old/new effect, whereas the other age groups did; and the shallow encoders showed an old/new effect, whereas the deep encoders did not.

There was no effect of depth (in contrast to the strong effect on accuracy) or interaction of age and depth $(\mathrm{F}=2.06, \mathrm{p}=.13)$.

\section{Naming}

Omissions or error rates for naming were very low $(<0.5 \%)$ as a result of the pretesting to ensure familiarity with the names. This involved a sample of the youngest age group's (the 5-year-olds) being asked to name the stimuli. Those not immediately named were rejected. Recall that the prediction for naming responses was faster latencies for old than for new stimuli, but no oldness $x$ depth interaction. Since memory based on physical characteristics should be independent of occurrence context, it should be insensitive to a levels manipulation.

Analyses of variance were performed on median naming latencies. The main oldness effect appeared as expected: All children named old stimuli $($ mean $=992 \mathrm{msec})$ faster than new (mean $=1,040 \mathrm{msec}$ ) $[\mathrm{F}(1,72)=31.17$, $\mathrm{MSe}=2,928, \mathrm{p}<.001]$. Increasing age reduced naming latencies, as might be expected $[\mathrm{F}(2,72)=49.96$, MSe $=38,173, p<.001]$, but there was no age $\times$ oldness interaction: All children named old stimuli faster than new, just as adults did in Experiment 1, and this was the case for all ages.

However, contrary to our prediction, there was an oldness $\times$ depth interaction $[\mathrm{F}(1,72)=4.50, \mathrm{MSe}=2,928$, $p<.05]$. This effect showed up at all ages, and thus there

Table 3

Median Correct Recognition Latencies (in Milliseconds), Experiment 3

\begin{tabular}{|c|c|c|c|c|}
\hline \multirow{3}{*}{$\begin{array}{l}\text { Age of } \\
\text { Subject }\end{array}$} & \multicolumn{4}{|c|}{ Depth of Encoding } \\
\hline & \multicolumn{2}{|c|}{ Shallow } & \multicolumn{2}{|c|}{ Deep } \\
\hline & Old & New & Old & New \\
\hline 5 -year-olds & 1756 & 1454 & 1800 & 1653 \\
\hline 7-year-olds & 1599 & 1354 & 1234 & 1229 \\
\hline 10-year-olds & 1065 & 1049 & 1037 & 1056 \\
\hline
\end{tabular}

Table 4

Mean Median Naming Latencies (in Milliseconds), Experiment 3

\begin{tabular}{ccccc} 
& \multicolumn{4}{c}{ Depth of Encoding } \\
\cline { 2 - 5 } Age of & \multicolumn{2}{c}{ Shallow } & \multicolumn{2}{c}{ Deep } \\
\cline { 2 - 5 } Subject & Old & New & Old & New \\
\hline 5-year-olds & 1170 & 1203 & 1220 & 1293 \\
7-year-olds & 970 & 998 & 950 & 1020 \\
10-year-olds & 845 & 875 & 797 & 854 \\
Average & 995 & 1025 & 898 & 1056 \\
\hline
\end{tabular}

was no three-way interaction $(F=.07$; see Table 4$)$. There is a much greater difference (i.e., $67 \mathrm{msec}$ ) between old and new naming times for deep stimuli than for shallow $(30 \mathrm{msec})$.

Our reason for including the response latencies for new items in the analyses was that experience taught us that there were considerable individual differences in speed among the children, especially the youngest ones, and treating as the dependent variable the difference between new and old times in a sense compensated for this. It is clear that it is only under this condition of analysis that depth has a significant effect. The means of the old items considered alone are $995 \mathrm{msec}$ for shallow and $989 \mathrm{msec}$ for deep, clearly not significantly different. The age $x$ depth interaction for old times also is not significant $[\mathrm{F}(2,72)=0.8]$.

We also calculated the $F$ ratios, using old and new items, for each age group separately. In no case was the interaction between old/new and shallow/deep significant. The $F$ values, from youngest to oldest, were $1.18,2.04$, and 1.65 , ( $\mathrm{df}=1,22$ in all cases; $\mathrm{p}>.10$ in all cases). The old/new effect was significant beyond .01 for each group considered separately.

\section{Discussion}

The recognition accuracy data show that developmental improvement in recognition (Sophian \& Stigler, 1981) survives obligatory encoding. Correct detection of old items was clearly dependent upon age despite the fact that all children were faced with one of two, fixed, orienting tasks. Thus, it seems likely that age-related changes in recognition skill cannot be attributed to the greater spontaneous use of "deep" encoding operations as children get older.

Of more immediate concern is the (usual) effect of depth upon recognition. All age groups did better after deep encoding than after shallow, mirroring the adult data from Experiment 1. Thus, we are free to make comparisons with the naming data, to check for dissociation. [ Incidentally, although recognition appears to improve with age with pictorial stimuli, sensitivity to depth does not. The youngest children were as affected by deep encoding, relative to the shallow group's performance, as were the other ages (the nonsignificant interaction term).]

The naming-latency results present something of an interpretation puzzle. First analysis fails to support the dis- 
sociation of recognition memory and perceptual memory in children, since both are susceptible to a depth-ofprocessing effect, in contrast to the situation for adults, for whom only recognition was so affected (Experiment 1). This is shown by the significant two-way interaction of depth and oldness. The reliability of this result, however, is open to question. It depends in part upon longer response times to new items for deep subjects at ages 5 and 7 (see Table 4), something that is not predicted by any theory. If new items are ignored, the deep/shallow manipulation is ineffective. It also requires the pooling of all subjects $(\mathrm{N}=78)$, since the interaction raiios fall well short of significance for each group treated separately. It is thus a relatively weak effect. This is confirmed by the fact that the value of $\Sigma$, a measure of strength of relationship, is only .21 .

Assuming for the moment that the effect is real, although not very strong, what could this mean? The straightforward interpretation is the one offered above: Recognition memory and perceptual memory are not different processes in children from 5 to 10 years of age. Nevertheless, we further pursued the question by attempting directly to assess perceptual memory, as described in the next experiment.

\section{EXPERIMENT 4}

In this study, we gave the usual deep and shallow orienting task to two groups of children (7-year-olds) and subsequently had them attempt to recognize old and new items presented very rapidly and in a somewhat degraded fashion. We were thus endeavoring to assess perceptual memory directly. It is known that, for adults, when words are used as stimuli, this technique demonstrates an old/ new effect but no effect due to depth of processing (Jacoby \& Dallas, 1981).

Method
Subjects
We wished to use children as young as was practicable in this
experiment to provide an effective endpoint in the development con-
tinuum. If dissociation could be demonstrated there, it would not
be necessary to test older children, since we now know that adults
also show independence between recognition and perceptual
memory. We chose 7 -year-olds rather than younger subjects be-
cause 5-year-olds tend to be more restless in this experimental sit-
uation and the nature of the task in Phase 2 (recognizing briefly
presented items) requires close attention. There were two groups
of children (14 in each group) selected from second grade classes
at one elementary school.

\section{Materials and Procedure}

The same slides were used as in the last experiment, 25 of them designated old in Phase 1. The two orienting tasks of searching for a cross and classifying in terms of portability were again used, with the usual counterbalancing across items to ensure that each one was as often old as new in Phase 2 . That phase consisted of the following: Each of the 50 pictures ( 25 old, 25 new) was projected for $80 \mathrm{msec}$ from a Kodak Carousel S-AV fitted with a Lafayette tachistoscopic shutter (Model 43015). Extensive pilot testing indicated a need to adjust aperture size to reduce the intensity of the picture in order to produce acceptable error rates. (Without a mask available, our pilot subjects produced almost error-free responses with a fully open aperture. The final adjustment chosen was approximately $3 \mathrm{~mm}$.) To familiarize them with the procedure in Phase 2, the children were first shown one picture for an extensive period (about $10 \mathrm{sec}$ ) with reduced brightness and then the same stimulus under tachistoscopic conditions. They were instructed to say what the object was, if possible; no emphasis was placed on speed of response. Then the 50 recognition items were presented. If any child gave indications that he or she knew what the object was but could not recall the name, he or she was probed and the item scored as correct if identification without naming had occurred (e.g., several subjects knew that the harp was used to make music but did not know its name) or if the name was retrieved upon probing. If after about $15 \mathrm{sec}$ no response had been made, or if the child said he/she had not seen the objects, a failure was recorded.

\section{Results}

The correct identification scores (proportion correct) for 14 subjects were: shallow encoding-old, .64, and new, .39; deep encoding-old, .65, and new, .39. Analysis of variance revealed only an old/new effect $[F(1,26)$ $=36.82, \mathrm{MSe}=125.7, \mathrm{p}<.001]$, and no effect due to depth $(F<1)$ or depth $\times$ old/new interaction.

\section{Discussion}

In contrast to recognition memory (Experiment 3 ), perceptual recognition is not affect by depth of processing at first exposure. What assists this form of memory is having seen the item previously (the oldness effect) and not what the subject has to do with it. This experiment resolves the ambiguity remaining after the previous experiment, in which depth did influence naming latency. Thus, children, like adults, show dissociation between recognition memory and perceptual memory.

\section{GENERAL DISCUSSION}

In Experiment 1, there was support for the distinction made by Jacoby and Dallas (1981) between two forms of memory in adults. These forms generalize across materials and tasks in adults. Specifically, a new test of perceptual memory, picture naming, produced results consistent with their account of this memory form: sensitivity only to physical characteristics (prior presentation) but not to meaningful (contextual) aspects of the stimulus. This interpretation was strengthened by Experiment 2, in essence a replication of the naming procedure in the first study.

The overall picture emerging from Experiments 3 and 4 was that in children, too, recognition memory and perceptual memory are separate processes. This was so despite the fact that the variable found useful in adults, naming latency, did show effects of depth of processing in children. This result may be a spurious one, but in any case a more direct test of perceptual memory, recognition under tachistoscopic conditions, failed to show a depth effect.

The fact of dissociation is perhaps most clearly demonstrated by the 5 -year-old children. The shallow recognition group successfully correctly recognized only about 
a quarter of the old items, but their counterparts in the naming condition nevertheless showed a substantial naming advantage for old items. Admittedly, this demonstration is not as dramatic as one using the same subjects for recognition and naming, but it is compelling nonetheless.

Perceptual memory, then, appears not to develop with age. This stands to reason, given its unaware property, but experimental evidence was needed.

We have also confirmed the equal sensitivity to encoding context at all ages in the recognition test, although recognition does appear to develop with age. But even very young children benefit from deep encoding when it is obligatory.

\section{REFERENCES}

Bower, G. H., \& KarLin, M. B. (1974). Depth of processing pictures of faces and recognition memory. Journal of Experimental Psychology, 103, 751-757.

Bransford, J. D., Nitsch, K. E., \& Franks, J. J. (1977). Schooling and the facilitation of knowing. In R. C. Anderson, R. J. Spiro, \& W. E. Montague (Eds.), Schooling and the acquisition of knowledge. Hillsdale, NJ: Erlbaum.

Brown, A. L. (1973). Judgments of recency for long sequences of pictures: The absence of a developmental trend. Journal of Experimental Child Psychology, 15, 473-480.

BRown, A. L. (1975). The development of memory: Knowing, knowing about knowing, and knowing how to know. In H. W. Reese (Ed.), Advances in child development and behavior (Vol. 10). New York: Academic Press.

Carroll, M., Gates, G. R., \& Roldan, F. (1984). Memory impairment in multiple sclerosis. Neuropsychologia, 22, 297-302.

Craik, F. I. M., \& Tulving, E. (1975). Depth of processing and the retention of words in episodic memory. Joumal of Experimental Psychology: General, 104, 268-294.

Flavell, J. H. (1977). Cognitive development. Englewood Cliffs, NJ: Prentice Hall.

Friedman, A., \& Bourne, L. E., JR. (1976). Encoding the levels of information in pictures and words. Journal of Experimental Psychology: General, 105, 169-190.

GeIS, M. F., \& HALL, D. M. (1976). Encoding and incidental memory in children. Journal of Experimental Child Psychology, 22, 58-66.

HASHER, L., \& ZACKS, R. T. (1979). Automatic and effortful processes in memory. Journal of Experimental Psychology: General, 108, 356-388

JACOBY, L. L., \& DALLAS, M. (1981). On the relationship between auto biographical memory and perceptual learning. Joumal of Experimental Psychology: General, 10, 306-340.

Mandler, J. A., \& Robinson, C. A. (1978). Developmental changes in picture recognition. Journal of Experimental Child Psychology, 26, 122-136.

NELSON, T. O. (1977). Repetition and depth of processing. Journal of Verbal Learning and Verbal Behavior, 16, 151-171.

OLsoN, G. M. (1976). An information processing analysis of visual memory and habituation in infants. In T. J. Tighe \& N. Leaton (Eds.), Habituation: Perspectives from child development, animal behavior, and neurophysiology. Hillsdale: NJ: Erlbaum.

Piaget, J., \& Inhelder, B. (1973). Memory and intelligence. New York: Basic Books.

SnOdgrass, J. C., \& Vanderwart, M. (1980). A standardized set of 260 pictures: Norms for name agreement, image agreement, familiarity, and visual complexity. Journal of Experimental Psychology: Human Learning and Memory, 6, 174-215.

SoPHIAN, C., \& STIGLER, J. W. (1981). Does recognition memory improve with age? Journal of Experimental Child Psychology, 32, 343-353.

(Manuscript received August 6, 1984; revision accepted for publication April 2, 1985.) 